\title{
Instability of the steady states of some Ginzburg-Landau-like equations with real coefficients
}

\author{
José M Vega \\ ETSI Aeronáuticos, Universidad Politécnica de Madrid, Plaza Cardenal Cisneros, 3. 28040 \\ Madrid, Spain
}

\begin{abstract}
The instability of the steady states with nonconstant amplitude is analysed for a nonlocal Ginzburg-Landau equation with real coefficients and quasiperiodic boundary conditions. The results are obtained in terms of easily recognized, qualitative properties of the steady states. Some of the results are new, even for the standard (local) Ginzburg-Landau equation with real coefficients. A related Ginzburg-Landau equation coupled to a mean field is also considered that appears in the analyses of counter-propagating waves in extended systems, nonoscillatory instabilities with a conservation law, and viscous Faraday waves in large aspect ratio containers.
\end{abstract}

Mathematics Subject Classification: 35B35, 35K20, 35G40, 76E30

\section{Introduction}

This paper deals with the stability of the steady states of several problems related to the standard Ginzburg-Landau equation,

$$
A_{t}=A_{x x}+\mu A-|A|^{2} A
$$

which appears as a universal equation near certain steady bifurcation points in extended systems $[1,2]$, in which the state variable $\boldsymbol{u}$ can be written as

$$
\boldsymbol{u}=\boldsymbol{u}_{0} A \mathrm{e}^{\mathrm{i} k x}+\text { c.c. }+\cdots,
$$

where $\boldsymbol{u}_{0}$ is an eigenfunction at marginal instability, and the complex amplitude $A$ depends weakly on both space and time. Equation (1.1) also appears in Floquet problems near marginal instability associated with the Floquet multiplier -1 ; in this case, the description (1.2) is still valid, but now the eigenfunction $\boldsymbol{u}_{0}$ is periodic in time. Equation (1.1) has received tremendous attention in the literature in the last 30 years. The coefficients of $A_{x x}$ and $|A|^{2} A$ have been set to one by rescaling $t, \mu$, and $A$, which requires that these two coefficients be real. 
The results below are new for equation (1.1) as well, but the main part of the paper (section 2) is concerned with the following nonlocal equation, with quasiperiodic boundary conditions:

$$
A_{t}=A_{x x}+\mu A-|A|^{2} A+\Gamma\left\langle|A|^{2}\right\rangle A, \quad A(x+1, t)=\mathrm{e}^{\mathrm{i} v} A(x, t),
$$

where $A$ is complex, the coefficients $\mu$ and $\Gamma$ are real, and $\langle\cdot\rangle$ denotes hereinafter the spatial average

$$
\langle f\rangle=\int_{0}^{1} f \mathrm{~d} x .
$$

$A$ is usually the complex amplitude of a slowly varying wavetrain in a periodic medium. The spatial period $L$ must be large compared to the wavelength of the basic wavetrain, and the spatial detuning $v$ measures the mismatch between both, namely $v=k L(\bmod 2 \pi)$, as obtained imposing spatial periodicity and invoking (1.2). Note that $v$ is generically nonzero if $L$ is (large but) finite, as is usually the case in practice, and can always be selected such that

$$
-\pi<v \leqslant \pi .
$$

The nonlocal problem (1.3) appears in a natural way [3-6] in the analysis of certain systems of coupled Ginzburg-Landau equations accounting for counter-propagating wavetrains [2], in the generic case in which the group velocity is of order one, and the length of the system is large but not too large [6]. But, in this case, the coefficients in (1.3a) are generally complex, and their being real is just a particular (codimension-four) case. There are cases in which equation (1.3a) is obtained with real coefficients from the outset: (i) in steady bifurcations of systems involving nonlocal terms from the outset (e.g. ferromagnetic resonance or current instability [7]), and (ii) in the analysis of standing, viscous Faraday waves [8-11] near threshold in appropriately large, two-dimensional, annular containers, as recently shown in [12]. Let us recall here that these waves appear in the surface of the liquid in a vertically vibrating container when the forcing acceleration exceeds a threshold value. The system exhibits a slowly varying (in both space and time) free surface deflection $f$ associated with a mean flow, whose evolution is coupled with that of the complex amplitude of the surface waves, $B$, according to (after appropriate scaling [12])

$$
\begin{aligned}
& B_{t}=B_{x x}+\mu B-\gamma_{0}|B|^{2} B-f B, \quad f_{t}=\gamma_{1} f_{x x}-\gamma_{2}\left(|B|^{2}\right)_{x x}, \\
& B(x+1, t)=\mathrm{e}^{\mathrm{i} v} B(x, t), \quad f(x+1, t)=f(x, t), \quad\langle f\rangle=0,
\end{aligned}
$$

where $\gamma_{0}, \gamma_{1}>0, \gamma_{2}$, and $f$ are real. Since this is a problem dealing with periodic waves, it may appear strange at first sight that the dynamics is described by the same equation as in steady bifurcation, but this is just a Floquet problem, with the instability setting in through the Floquet multiplier -1 , as in the simpler case explained after equation (1.2). In some limits (e.g. if viscosity is somewhat small, but not too small, in order to avoid a more complex structure of both the amplitude equations $[13,14]$ and the mean flow $[15,16]), \gamma_{1}$ and $\gamma_{2}$ are large, and the free surface deflection becomes slaved to surface waves as

$$
f=\frac{\gamma_{2}\left(|B|^{2}-\left\langle|B|^{2}\right\rangle\right)}{\gamma_{1}},
$$

where the nonlocal term results from volume conservation $(1.5 c)$. Substitution of (1.6) into (1.4) leads to (1.3) after re-scaling if $\gamma_{0} \gamma_{1}+\gamma_{2}>0$; if instead $\gamma_{0} \gamma_{1}+\gamma_{2}<0$, then we obtain (cf (1.3))

$$
A_{t}=A_{x x}+\mu A+|A|^{2} A-\Gamma\left\langle|A|^{2}\right\rangle A, \quad A(x+1, t)=\mathrm{e}^{\mathrm{i} v} A(x, t) .
$$


This problem is also of interest because it exhibits nontrivial exponentially stable steady states if $\Gamma>1$ (see below). Equations (1.4)-(1.5) apply to various pattern formation problems [17-19], and similar Ginzburg-Landau equations coupled to a mean field appear in, e.g., BénardMarangoni convection [20,21]. A mean field also appears in, e.g., convection in rotating annuli [22], and related (but different) nonlocal Ginzburg-Landau equations are also obtained in reaction-diffusion systems [23].

The steady states of (1.3) are calculated in terms of elliptic functions [18], but the interest of these is limited unless they are stable and checking this is not trivial. The main object of this paper is to obtain useful stability results in terms of easily recognized properties of the steady states. In particular, we shall prove that if $\Gamma \geqslant 0$ then all steady states of (1.3) such that $|A|$ is nonconstant are unstable (theorem 2.14). This is the main result of the paper. This property (which has been said to be 'well-known' by heuristic arguments [24]) solves an open problem for the standard, local $(\Gamma=0)$ Ginzburg-Landau equation with real coefficients in a (large but) bounded domain. A related result that applies in an unbounded domain has been rigorously proved by Doelman et al [25]. They considered spatially quasiperiodic steady states of (1.1) and imposed an independent spatial quasiperiodicity condition on the linearized eigenvalue problem associated with linear stability. In our notation, the latter was posed by equations (2.9)-(2.10) below (with $\Gamma=0$ ), but with boundary conditions (cf (2.11))

$$
X(x+1)=\gamma X(x) \mathrm{e}^{\mathrm{i} v}, \quad Y(x+1)=\bar{\gamma} Y(x) \mathrm{e}^{-\mathrm{i} v},
$$

where $\gamma \in \mathbb{C}$ is such that $|\gamma|=1$. The eigenvalues of (1.8), (2.9)-(2.10) were called $\gamma$-eigenvalues in [25] and the main result was that if $\left|A_{s}\right|$ is not constant and $\gamma \neq 1$, then there is a strictly positive (real) $\gamma$-eigenvalue. This implies that the steady state is unstable in the laterally unbounded case, but not as a solution of (1.1) with boundary conditions $(1.3 \mathrm{~b})$ because $\gamma \neq 1$. The result does not apply to the case $\gamma=1$, and neither can it be extended to this case in an obvious way [25, pp 513-4] because the proof essentially relies on the property that $\lambda=0$ is not an eigenvalue, which is untrue if $\gamma=1$ (see below). The stability of some steady states of (1.4)-(1.5), with $\nu=0, B$ real (quite a strong restriction that requires that the length of the spatial domain be commensurable with the wavelength of the basic wave, which is nongeneric and greatly simplifies the analysis) has been recently analysed by Norbury et al [26]; in addition, they considered the limit $|\mu| \gg 1$, which with their nondimensionalization corresponds to $L \gg 1$.

The analysis below relies on the fact that the coefficients are real. This will allow a not too sophisticated proof of the main results, but these are still nontrivial because

(a) The system of real equations obtained from (1.3) for the real and imaginary parts of the complex amplitude $A$ is not cooperative. Thus, maximum principles cannot be used, and standard results ensuring that the eigenfunctions associated with the most dangerous eigenvalue have a constant sign do not apply. The latter property is essential in the proof of classical results on the instability of nonconstant steady states of real second-order parabolic equations with periodic boundary conditions.

(b) $\lambda=0$ is always an eigenvalue of the linearized problem (see below). Thus, topological continuation methods, based on the invariance of the number of eigenvalues in a domain $\Sigma$ of the complex plane $(\Sigma: \Re(\lambda)>0$ in the present context) under appropriate transformations, must be handled with care because this argument requires the absence of eigenvalues in the boundary of $\Sigma$. The presence of the zero eigenvalue instead will be an advantage, as will be seen below.

Using standard results on semi-linear parabolic problems [27, theorem 3.3.4, pp 55-6] (in conjunction with local parabolic estimates [28] and imbedding theorems [29]) we readily 
see that for appropriate initial conditions, the problems (1.3), (1.4)-(1.5), and (1.7) possess a unique classical solution in a maximal interval of existence $0<t<T \leqslant \infty$, and that either $T=\infty$ or the solution diverges as $t \nearrow T$; also, standard estimates show that the former alternative holds for (1.3) if $\Gamma<1$ and for (1.4)-(1.5) if $\left(\gamma_{1}>0\right.$ and) $\gamma_{1}+\gamma_{2}>0$. The three problems admit a Lyapunov function, as seen from the exact relations

$$
\frac{\mathrm{d}}{\mathrm{d} t}\left[\left\langle\left|A_{x}\right|^{2}\right\rangle-\mu\left\langle|A|^{2}\right\rangle \pm \frac{\left\langle|A|^{4}\right\rangle-\Gamma\left\langle|A|^{2}\right\rangle^{2}}{2}\right]=-2\left\langle\left|A_{t}\right|^{2}\right\rangle,
$$

where the + and - signs apply for (1.3) and (1.7), respectively. For (1.4)-(1.5), the relevant relation is

$$
\frac{\mathrm{d}}{\mathrm{d} t}\left[\left\langle\left|B_{x}\right|^{2}\right\rangle-\mu\left\langle|B|^{2}\right\rangle+\frac{\gamma_{0}\left\langle|B|^{4}\right\rangle}{2}-\frac{\gamma_{1}\left\langle\phi_{x}^{2}\right\rangle}{2 \gamma_{2}}+\left\langle|B|^{2} \phi_{x}\right\rangle\right]=-2\left\langle\left|B_{t}\right|^{2}\right\rangle+\frac{\left\langle\phi_{t}^{2}\right\rangle}{\gamma_{2}}
$$

with $\phi=\int_{0}^{x} f(\eta, t) \mathrm{d} \eta$, and defines a Lyapunov function provided that $\gamma_{2}<0$. Thus, using standard results on infinite dimensional (gradient) dynamical systems [30, p 50, lemma 3.8.2], we see that the $\omega$-limit set of the bounded orbits of the dynamical systems associated with (1.3) and (1.7) is contained in the set of steady states; the same is true for (1.4)-(1.5) provided that $\gamma_{2}<0$.

The problems (1.3) and (1.7) are invariant under the actions

$$
\begin{aligned}
& \left.x \rightarrow x+c_{1} \quad \text { (for all } c_{1} \in \mathbb{R}\right) ; \quad A \rightarrow A \mathrm{e}^{\mathrm{i} c_{2}} \quad\left(\text { for all } c_{2} \in \mathbb{R}\right) ; \\
& x \rightarrow-x ; \quad \text { and } \quad A \rightarrow \bar{A} .
\end{aligned}
$$

These result from invariance under spatial translations and reflection of the original problem from which (1.3) and (1.7) have been derived, and will play an important role below. Similarly, (1.4)-(1.5) are invariant under the actions

$$
\begin{aligned}
& \left.x \rightarrow x+c_{1} \quad\left(\text { for all } c_{1} \in \mathbb{R}\right) ; \quad B \rightarrow B \mathrm{e}^{\mathrm{i} c_{2}} \quad \text { (for all } c_{2} \in \mathbb{R}\right) ; \\
& x \rightarrow-x ; \quad \text { and } \quad B \rightarrow \bar{B} .
\end{aligned}
$$

Because of these, the steady states are not isolated. For each steady state of (1.3) and (1.7) (resp., (1.4)-(1.5)) such that $|A|$ (resp., $|B|$ ) is nonconstant, these actions generate a whole 2-torus of steady states; if $|A|$ (resp., $|B|$ ) is constant, the actions still generate a circle of steady states. But generically we expect that these 2 -tori and circles will be isolated. In this case, we can apply [27, pp 108-9 and pp 174-5] to show that all bounded solutions converge to steady states as $t \rightarrow \infty$. Thus, excluding unbounded solutions, the large time dynamics of the problems (1.3), (1.7), and (1.4)-(1.5) are dominated by the steady states, provided that $\gamma_{2}<0$.

The remainder of this paper is organized as follows. Several linear stability results on (1.3) are obtained in section 2, where in addition to the main result (theorem 2.14), which applies for $\Gamma \geqslant 0$, we ensure instability of the steady states with nonconstant $|A|$, without any restriction on the sign of $\Gamma$ provided that (i) $|A|$ is not too large and the phase of $A$ increases more than $\pi$ in each period (proposition 2.7), or (ii) the phase of $A$ either is constant or does not vary much (proposition 2.8), which in particular contains all instability results in [26]), or (iii) the function $x \rightarrow|A(x)|$ exhibits at least two maxima in each period (proposition 2.10). For completeness, the stability properties of the spatially constant steady states of (1.3) (already obtained by Elmer [7]) are also considered in proposition 2.3. The counterparts of these results for equation (1.7) and the system (1.4)-(1.5) are obtained in sections 3 and 4, respectively, and some concluding remarks are made in section 5 . 


\section{Steady states of (1.3) and their linear stability}

The nontrivial ( $A_{s}$ not identically zero) steady states of (1.3) satisfy

$A_{s}^{\prime \prime}+\mu A_{s}-\left|A_{s}\right|^{2} A_{s}+\Gamma\left\langle\left|A_{s}\right|^{2}\right\rangle A_{s}=0, \quad A_{s}(x+1)=\mathrm{e}^{\mathrm{i} \nu} A_{s}(x)$,

and can be written as

$$
A_{s}=R_{s}(x) \mathrm{e}^{\mathrm{i} \theta_{s}(x)},
$$

where $R_{s}$ and $\theta_{s}$ are given by

$$
\begin{aligned}
& R_{s}^{\prime \prime}-R_{s} \theta_{s}^{\prime 2}+\mu R_{s}-R_{s}^{3}+\Gamma\left\langle R_{s}^{2}\right\rangle R_{s}=0, \quad R_{s} \theta_{s}^{\prime \prime}+2 R_{s}^{\prime} \theta_{s}^{\prime}=0, \\
& R_{s}(x+1)=R_{s}(x), \quad \theta_{s}(x+1)=\theta_{s}(x)+v(\bmod 2 \pi) .
\end{aligned}
$$

From these equations we readily obtain the following properties, which are collected here for convenience.

Lemma 2.1. The nontrivial steady states of (1.3) are analytic and such that:

(i) If $A_{s}\left(x_{1}\right)=0$ then $A_{s}^{\prime}\left(x_{1}\right)=\left[R_{s}^{\prime}\left(x_{1}\right)+\mathrm{i} R_{s}\left(x_{1}\right) \theta_{s}^{\prime}\left(x_{1}\right)\right] \mathrm{e}^{\mathrm{i} \theta_{s}\left(x_{1}\right)} \neq 0$.

(ii) Each solution is such that either (a) $\theta_{s}=$ constant (which requires that either $v=0$ or $\pi$ ), with $R_{s}$ possibly not of constant sign, or $(b)$ both $\theta_{s}^{\prime} \neq 0$ and $R_{s} \neq 0$ for all $x$. In the second case, we have

$$
\begin{aligned}
& R_{s}^{\prime \prime}-c^{2} R_{s}^{-3}+\mu R_{s}-R_{s}^{3}+\Gamma\left\langle R_{s}^{2}\right\rangle R_{s}=0, \quad R_{s}(x+1)=R_{s}(x), \\
& \theta_{s}=c \int_{0}^{x} R_{s}^{-2} \mathrm{~d} x+\text { constant }
\end{aligned}
$$

where the constant $c$ is given by

$$
c=\frac{\nu+2 n \pi}{\left\langle R_{s}^{-2}\right\rangle}, \quad \text { with } n=0, \pm 1, \pm 2, \ldots
$$

(iii) If $R_{s}^{\prime}\left(x_{1}\right)=0$, then the steady state is reflection-symmetric around $x_{1}$, namely (see (1.9))

$$
R_{s}\left(x_{1}-z\right)=R_{s}\left(x_{1}+z\right), \quad \theta_{s}\left(x_{1}-z\right)=2 \theta_{s}\left(x_{1}\right)-\theta_{s}\left(x_{1}+z\right) \quad \text { for all } z \in \mathbb{R} .
$$

Proof. $A_{s}$ is analytic because its real and imaginary parts are solutions of a system of ODEs with analytic coefficients [31].

Property (i) follows because if both $A_{s}$ and $A_{s}^{\prime}$ are zero at $x_{1}$, then $A_{s}$ is identically zero by uniqueness of the Cauchy problem posed by $(2.1 a)$ with initial conditions $A_{s}\left(x_{1}\right)=A_{s}^{\prime}\left(x_{1}\right)=0$.

In order to prove (ii) note that, according to equation $(2.3 b)$, if $R_{s} \neq 0$ and $\theta_{s}^{\prime} \neq 0$ for some $x_{0} \in \mathbb{R}$, then, in an interval $I$ including $x_{0}$ we have

$$
R_{s}^{2} \theta_{s}^{\prime}=c
$$

for some constant $c \neq 0$, as obtained upon integration of (2.3b). Also, obviously, either we can take $I=\mathbb{R}$ or one of the end-points of $I$, say $x_{0}$, is such that $R_{s} \rightarrow 0$ as $x \rightarrow x_{0}$; but the second alternative requires that $\theta_{s}^{\prime}=c / R_{s} \rightarrow \infty$ as $x \rightarrow x_{0}$, which cannot happen because the function $x \rightarrow A_{s}(x)$ is analytic. Thus, $R_{s} \neq 0$ and $\theta_{s}^{\prime} \neq 0$ are well defined in $\mathbb{R}$, and using (2.3) and (2.6) we readily obtain (2.4) and (2.5).

Finally, property (iii) follows by uniqueness of the Cauchy problem posed by $(2.1 a)$, with initial data at $x=x_{1}$, and invariance of (2.1) under (1.9). This completes the proof. 

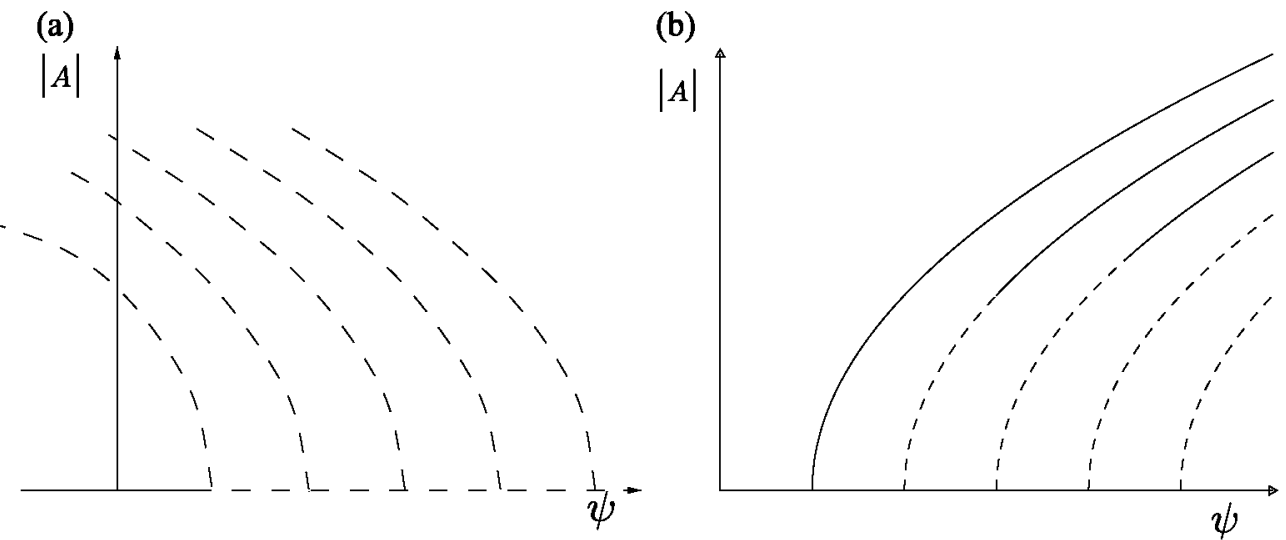

Figure 1. Unstable (- - -) and stable (-) spatially uniform SWs of (1.3) for: $(a) \Gamma>1$ and (b) $\Gamma<1$. Plot (b) also applies to the system (1.4)-(1.5) (replacing $A$ by $B$ ) provided that $\gamma_{2}<0$ and $\gamma_{0} \gamma_{1}+\gamma_{2}>0$.

Remark 2.2. Property (iii) implies that if $R_{S}$ is not constant, and exhibits two consecutive maxima at $x=x_{1}$ and $x_{1}+\ell$, then the function $R_{s}$ exhibits two minima at $x=x_{1} \pm \ell / 2$, and is $\ell$-periodic.

The trivial steady state of (1.3), $A_{s}=0$, is exponentially stable if and only if $\mu<v^{2}$. There are also steady states with constant amplitude, of the form

$$
A_{s}=R_{n} \mathrm{e}^{\mathrm{i}(v+2 n \pi) x}, \quad \text { for } n=0, \pm 1, \pm 2, \ldots
$$

with $R_{n}>0$ given by

$$
\mu=(v+2 n \pi)^{2}+(1-\Gamma) R_{n}^{2} .
$$

These are in branches that bifurcate from the trivial branch at $\mu=(\nu+2 n \pi)^{2}$, supercritically if $\Gamma<1$ and sub-critically if $\Gamma>1$ (see figure 1). In addition, there are steady states with nonconstant amplitude. These do not bifurcate from the trivial branch if $v \neq 0, \pi$; if $v=0$ (resp., $v=\pi$ ) instead there are infinitely many branches of steady states with nonconstant amplitude and constant phase that bifurcate from the trivial branch at $\mu=(2 n \pi)^{2}$ (resp., $\left.\mu=(2 n-1)^{2} \pi^{2}\right)$. These statements follow by a standard local bifurcation analysis.

The linear stability of a nontrivial steady state $A_{s}$ is analysed as usual, replacing $A-A_{s}$ by $X(x) \mathrm{e}^{\lambda t}+\bar{Y}(x) \mathrm{e}^{\bar{\lambda} t}$ in (1.3) and linearizing, to obtain

$$
\begin{aligned}
& \lambda X=X^{\prime \prime}+\left(\mu+\Gamma\left\langle\left|A_{s}\right|^{2}\right\rangle-2\left|A_{s}\right|^{2}\right) X-A_{s}^{2} Y+\Gamma\left\langle\bar{A}_{s} X+A_{s} Y\right\rangle A_{s}, \\
& \lambda Y=Y^{\prime \prime}+\left(\mu+\Gamma\left\langle\left|A_{s}\right|^{2}\right\rangle-2\left|A_{s}\right|^{2}\right) Y-\bar{A}_{s}^{2} X+\Gamma\left\langle\bar{A}_{s} X+A_{s} Y\right\rangle \bar{A}_{s}, \\
& X(x+1)=X(x) \mathrm{e}^{\mathrm{i} v}, \quad Y(x+1)=Y(x) \mathrm{e}^{-\mathrm{i} v} .
\end{aligned}
$$

This problem is solved in closed form if $A_{s}$ is as given in (2.7)-(2.8). In this case, the eigenfunctions are of the form $X=X_{0} A_{s} \mathrm{e}^{2 \mathrm{i} m \pi x}$ and $Y=Y_{0} \bar{A}_{s} \mathrm{e}^{2 \mathrm{i} m \pi x}$, where the wavenumber $m$ must be an integer, and $X_{0}$ and $Y_{0}$ are complex constants. The dispersion relation is

$$
\begin{aligned}
& {\left[\lambda+(1-\Gamma) R_{n}^{2}\right]^{2}=(1-\Gamma)^{2} R_{n}^{4} \quad \text { if } m=0,} \\
& \left(\lambda+4 m^{2} \pi^{2}+R_{n}^{2}\right)^{2}=16 m^{2} \pi^{2}(v+2 n \pi)^{2}+R_{n}^{4} \quad \text { if } m \neq 0 .
\end{aligned}
$$


Note that $\lambda=0$ is always an eigenvalue (for $m=0$ ), but this must be excluded in the stability analysis because it results from invariance under $(1.9 a)$. The following result was already obtained by Elmer [7].

Proposition 2.3. If $\Gamma>1$ then all steady states of the form (2.7) are exponentially unstable. If $\Gamma<1$ then the steady states (2.7) are: (i) orbitally, exponentially stable if either $n=0$ or $n \neq 0$ and

$$
R_{n}^{2}>2(v+2 n \pi)^{2}-2 \pi^{2}
$$

and (ii) exponentially unstable if $n \neq 0$ and the opposite inequality holds (see figure 1).

Proof. The (always real) eigenvalue $\lambda$ is obtained in closed form from (2.12), and the stated result is readily checked.

In fact, the eigenvalues of (2.9)-(2.11) are all real also for the remaining steady states, with nonconstant $\left|A_{s}\right|$. This is seen from the following equation:

$$
\begin{aligned}
\lambda\left\langle|X|^{2}+|Y|^{2}\right\rangle & =-\left\langle\left|X^{\prime}\right|^{2}+\left|Y^{\prime}\right|^{2}\right\rangle+\left\langle\left(\mu+\Gamma\left\langle\left|A_{s}\right|^{2}\right\rangle-2\left|A_{s}\right|^{2}\right)\left(|X|^{2}+|Y|^{2}\right)\right\rangle \\
& -\left\langle A_{s}^{2} \bar{X} Y+\text { c.c. }\right\rangle+\Gamma\left|\left\langle\bar{A}_{s} X+A_{s} Y\right\rangle\right|^{2},
\end{aligned}
$$

which is obtained (exploiting the fact that (2.7)-(2.8) is self-adjoint with the inner product of $L_{2} \times L_{2}$ ) by multiplying (2.9) by $\bar{X}$ and (2.10) by $\bar{Y}$, adding, integrating in $0<x<1$, integrating by parts, and substituting (2.11). Since $\lambda$ is real, we can add (2.9) to the complex conjugate of (2.10), add (2.11a) to the complex conjugate of (2.11b), and substitute $X+\bar{Y}$ by $X$, to reduce (2.9)-(2.11) to the simpler eigenvalue problem

$$
\begin{aligned}
& \lambda X=X^{\prime \prime}+\left(\mu+\Gamma\left\langle\left|A_{s}\right|^{2}\right\rangle-2\left|A_{s}\right|^{2}\right) X-A_{s}^{2} \bar{X}+\Gamma\left\langle\bar{A}_{s} X+A_{s} \bar{X}\right\rangle A_{s}, \\
& X(x+1)=X(x) \mathrm{e}^{\mathrm{i} \nu} .
\end{aligned}
$$

Conversely, if (2.14)-(2.15) hold then $X$ and $Y=\bar{X}$ satisfy (2.9)-(2.11). Thus, the spectra of (2.9)-(2.11) and (2.14)-(2.15) coincide.

\section{Remarks 2.4.}

(A) The eigenspaces of (2.14)-(2.15) are spanned by a set of linearly independent eigenfunctions with real coefficients. In fact, because of the term proportional to $\bar{X}$, the eigenvalue problem (2.14)-(2.15) is not linear in a standard complex function space. This problem must be treated in the 'real' function spaces obtained when the sets of complex valued functions $C^{k}$ and $H^{k}$ are considered as vector spaces over the field $\mathbb{R}$ (instead of $\mathbb{C})$. The eigenfunctions associated with different eigenvalues are orthogonal to each other with the 'real' inner product

$$
\left(X_{1}, X_{2}\right)=\frac{1}{2} \int_{0}^{1}\left(\bar{X}_{1} X_{2}+\text { c.c. }\right) \mathrm{d} x .
$$

(B) If $A_{s}$ is a nontrivial steady state of (1.3) then $\lambda=0$ is always an eigenvalue, with eigenfunctions

$$
X=A_{s}^{\prime} \quad \text { (if } A_{s} \neq \text { constant) and } \quad X=\mathrm{i} A_{s},
$$

which result from invariance of the original problem under the actions $(1.9 a)$ and $(1.9 b)$. If $\left|A_{s}\right|$ is constant then the eigenfunctions (2.16) are proportional to each other, but otherwise they are linearly independent. 
The eigenfunctions (2.16) and invariance under (1.9) must be taken into account to obtain the following stability result:

Lemma 2.5. If the zero eigenvalue of (2.14)-(2.15) has no other eigenfunctions than (2.16), and the remaining eigenvalues are negative, then the steady state $A_{s}$ is locally, 'orbitally, exponentially stable', with 'asymptotic phase'. If the system (2.14)-(2.15) has a strictly positive eigenvalue, then the steady state is locally, exponentially unstable.

Proof. This result follows from [27, pp 108-9 and pp 174-5].

Now, standard results on compact, self-adjoint operators and a standard variational argument [32-34] show that the eigenvalues of (2.14)-(2.15) are such that (cf (2.13)) $\lambda\left\langle|X|^{2}\right\rangle=-\mathcal{A}(X)$, where the quadratic functional $\mathcal{A}$ is defined as

$\mathcal{A}(X)=\left\langle\left|X^{\prime}\right|^{2}\right\rangle-\left\langle\left(\mu+\Gamma\left\langle\left|A_{s}\right|^{2}\right\rangle-2\left|A_{s}\right|^{2}\right)|X|^{2}\right\rangle+\frac{\left\langle A_{s}^{2} \bar{X}^{2}+\text { c.c. }\right\rangle-\Gamma\left(\bar{A}_{s} X+\text { c.c. }\right\rangle^{2}}{2}$

and the maximum eigenvalue is given by

$\lambda_{M}=-\min \left\{\frac{\mathcal{A}(X)}{\left\langle|X|^{2}\right\rangle}: 0 \neq X \in H^{1}, X(x+1)=\mathrm{e}^{\mathrm{i} v} X(x)\right.$ for all $\left.x\right\}$.

The minimizers are precisely the eigenfunctions associated with $\lambda_{M}$, and are analytic even though the minimum in (2.18) is taken in $H^{1}$. These are the key ingredients to prove the instability results below.

Lemma 2.6. Let $A_{s}$ be a nontrivial solution of (2.1), and let $X$ be a function of $H^{1}$ such that $X(x+1)=\mathrm{e}^{\mathrm{i} v} X(x)$ for all $x$, and either $(i) \mathcal{A}(X)<0$, or (ii) $X$ is not an eigenfunction of (2.14)-(2.15) (which happens in particular if $X$ is not analytic) and $\mathcal{A}(X)=0$. Then (2.14)-(2.15) has a strictly positive eigenvalue.

Proof. This is a straightforward consequence of the facts that (a) (2.18) provides the largest eigenvalue of (2.14)-(2.15), and (b) the minimizers in (2.18) are the eigenfunctions associated with $\lambda_{M}$.

Using this, we first extend a part of the instability result in proposition 2.3 to steady states with nonconstant $\left|A_{s}\right|$ : if $n \neq 0$ and $\left|A_{s}\right|$ is sufficiently small, then the steady state is exponentially unstable.

Proposition 2.7. Shift $x$ so that $R_{s}$ is an even function of $x$ (which according to lemma 2.1(iii) is always possible). Let the integer $n$, defined in (2.5), be such that $|v+2 n \pi|>\pi$. Then, the steady states defined by (2.2), (2.4) and (2.5), with $R_{s} \neq 0$ for all $x$, are exponentially unstable provided that

$$
\left\langle R_{s}^{4}(1-\cos 2 \pi x)\right\rangle \leqslant 4 \pi\left(\frac{|\nu+2 n \pi|}{\left\langle R_{s}^{-2}\right\rangle}-\pi\left\langle R_{s}^{2}\right\rangle\right) .
$$

Proof. Let $X=\mathrm{i} R_{s} \mathrm{e}^{\mathrm{i}\left(\theta_{s}+2 m \pi x\right)}$ and $Y=\mathrm{i} R_{s} \mathrm{e}^{\mathrm{i} \theta_{s}}$, with $m=-(v+2 n \pi) /|v+2 n \pi|(= \pm 1)$. Since $Y$ is an eigenfuction of (2.14)-(2.15) associated with the zero eigenvalue (remark 2.4(B)), according to our comments above, we have $\mathcal{A}(Y)=0$. But, also, invoking the definition (2.17) we obtain, after some algebra,

$-\mathcal{A}(X)=\mathcal{A}(Y)-\mathcal{A}(X)=\left\langle R_{s}^{2}\left[\theta_{s}^{\prime 2}-\left(\theta_{s}^{\prime}+2 m \pi\right)^{2}\right]\right\rangle-\left\langle R_{s}^{4}(1-\cos 4 m \pi x)\right\rangle$, 
where we have taken into account that $R_{s}$ and $\cos 2 m \pi x$ are even in $x$, and $\sin 2 m \pi x$ is odd. But

$$
\begin{aligned}
\left\langle R_{s}^{2}\left[\theta_{s}^{2}-\left(\theta_{s}^{\prime}+2 m \pi\right)^{2}\right]\right\rangle & =\left\langle R_{s}^{2}\left[-4 m \pi \theta_{s}^{\prime}-(2 m \pi)^{2}\right]\right\rangle=-4 m \pi c-\left\langle R_{s}^{2}\right\rangle(2 m \pi)^{2} \\
& =\frac{4 \pi|\nu+2 n \pi|}{\left\langle R_{s}^{-2}\right\rangle}-4 \pi^{2}\left\langle R_{s}^{2}\right\rangle
\end{aligned}
$$

as obtained invoking (2.5), (2.6), and the definition of $m$ above. Using this, (2.19) and (2.20), we obtain $\mathcal{A}(X)<0$ and we only need to apply lemma 2.6 to obtain the stated result. Thus, the proof is complete.

The remaining part of the instability result in proposition 2.3 is extended in the following proposition.

Proposition 2.8. A steady state of (1.3) is unstable if $\left|A_{s}\right|$ is not constant and either:

(i) The phase of $A_{s}$ is constant (namely, $A_{s}=R_{s} \mathrm{e}^{\mathrm{i} \theta_{0}}$, with $R_{s}$ not necessarily of constant sign and $\theta_{0}=$ constant, which requires that either $v=0$ or $\pi$ ), or

(ii) $A_{s}=R_{s} \mathrm{e}^{\mathrm{i} \theta_{s}}$, with $\theta_{s}$ nonconstant, and $\left.\left\langle R_{s}^{2} R_{s}^{2}\right\rangle+(\Gamma-1)\left[\theta_{s}(1)-\theta_{s}(0)\right]^{2} /\left\langle R_{s}^{-2}\right\rangle^{2}\right\rangle 0$.

Proof. Let us first see that all steady states are unstable if

$$
\left\langle A_{s}^{2} \bar{A}_{s}^{\prime 2}+\text { c.c. }\right\rangle-\frac{\Gamma}{2}\left\langle\bar{A}_{s} A_{s}^{\prime}-\text { c.c. }\right\rangle^{2}>0 \text {. }
$$

To this end, we apply lemma 2.6, taking into account that since $Y=A_{s}^{\prime}$ is an eigenfunction of (2.14)-(2.15) associated with the zero eigenvalue (remark $2.4(\mathrm{~B})$ ), we have $\mathcal{A}(Y)=0$, and that $X=\mathrm{i} Y$ satisfies

$$
\mathcal{A}(X)=\mathcal{A}(X)-\mathcal{A}(Y)=-\left\langle A_{s}^{2} \bar{A}_{s}^{\prime 2}+\text { c.c. }\right\rangle+\frac{\Gamma}{2}\left\langle\bar{A}_{s} A_{s}^{\prime}-\text { c.c. }\right\rangle^{2}<0 .
$$

The stated result follows because condition (2.21) holds under any of the assumptions stated in cases (i) and (ii), as is readily seen using (2.2) and (2.6). This completes the proof.

Remark 2.9. This result implies that a steady state with nonconstant $\left|A_{s}\right|$ is unstable in the following cases:

(a) If the phase of $A_{s}$ is constant, as is the case for all steady states of (1.3) calculated in [18] (plotted in [18, figure 7]). Instability of these patterns in the limit $|\mu| \gg 1$ was obtained in [26].

(b) If $\Gamma<1$ but the phase of $A_{s}$ does not vary much.

(c) If $\Gamma \geqslant 1$, without further restrictions.

The following result implies that if $\left|A_{s}\right|$ oscillates more than once in the period $0<x \leqslant 1$, then the steady state is unstable.

Proposition 2.10. Let $A_{s}$ be a steady state of (2.2) such that $\left|A_{s}(x)\right|$ is not constant and exhibits more than one local maximum in the interval $0 \leqslant x<1$. Then, $A_{s}$ is exponentially unstable.

Proof. Shift $x$ such that two consecutive maxima are attained at $x=0$ and $x=\ell<1$. According to remark 2.2, both $R_{s}$ and $\theta_{s}^{\prime}$ are periodic of period $\ell$, and (see equation (2.6))

$$
R_{s}(0)=R_{s}(\ell), \quad R_{s}^{\prime}(0)=R_{s}^{\prime}(\ell)=0, \quad \theta_{s}^{\prime}(0)=\theta_{s}^{\prime}(\ell) .
$$

Also, the functions (2.16) are eigenfunctions of $(2.14)-(2.15)$ associated with the zero eigenvalue. Thus,

$$
Y=A_{s}^{\prime}-\mathrm{i} \theta_{s}^{\prime}(0) A_{s}
$$


is also an eigenfunction associated with the zero eigenvalue, and since $A_{s}^{\prime}=\left(R_{s}^{\prime}+\mathrm{i} R_{s} \theta_{s}^{\prime}\right) \mathrm{e}^{\mathrm{i} \theta_{s}}$, according to (2.22) we have $Y(0)=Y(\ell)=0$. Then, the function $X$, defined as $X=Y$ if $0 \leqslant x \leqslant \ell$ and $X=0$ if $\ell<x<1$ and extended to the whole line $\mathbb{R}$ by means of (2.15), is in $H^{1}$ but is not analytic, and satisfies

$$
\mathcal{A}(X)=0
$$

This is seen by multiplying the eigenvalue equation (2.14) (with $\lambda=0$ ) by $\bar{X}$, integrating in $0<x<\ell$, integrating by parts, and taking into account that $X(0)=X(\ell)=0$ and $\left\langle\bar{A}_{s} X+\right.$ c.c. $\rangle=\left\langle\bar{A}_{s} A_{s}^{\prime}+\right.$ c.c. $\rangle=\left|A_{s}(\ell)\right|^{2}-\left|A_{s}(0)\right|^{2}=0$. Thus, lemma 2.6 yields the stated result, and the proof of the theorem is complete.

As a straightforward consequence of the result above, we have the following generalization of the main result in [25] to the nonlocal equation (1.3a), concerning the quasiperiodic steady states (such that $\left|A_{s}\right|$ is nonconstant and periodic) of the laterally unbounded problem

$A_{t}=A_{x x}+\mu A-\left[|A|^{2}-\Gamma\left\langle|A(y, t)|^{2}\right\rangle\right] A \quad$ in $-\infty<x<\infty$

with

$$
\left\langle|A|^{2}\right\rangle \equiv \lim _{L \rightarrow \infty} \frac{1}{2 L} \int_{-L}^{L}|A|^{2} \mathrm{~d} x=\text { bounded. }
$$

Corollary 2.11. Any (quasiperiodic) steady state of the initial value problem (2.23) such that $\left|A_{s}\right|$ is periodic and nonconstant, is exponentially unstable.

Proof. The period of $A_{s}$ may be set to $\frac{1}{2}$ by rescaling $x$ (and also rescaling $t, A$ and $\mu$, in order to leave equation (1.3) invariant). Then $\left|A_{s}\right|$ exhibits at least two maxima in $0 \leqslant x<1$ and according to proposition $2.10, A_{s}$ is exponentially unstable as a steady state of (1.3). And since all solutions of (1.3) satisfy (2.23)-(2.24), the result follows.

According to proposition 2.8, all solutions are unstable if $\Gamma \geqslant 1$, but we can obtain the same conclusion for $\Gamma \geqslant 0$ (which includes the standard, local Ginzburg-Landau equation for $\Gamma=0$ ). The main idea to obtain this result is as follows. Since the eigenvalues are real, all instabilities are associated with bifurcations to steady states, which are of the form (2.2), with $R_{s}$ and $\theta_{s}$ given by (2.4). Thus, we expect that (2.14)-(2.15) possess the zero eigenvalue if and only if the linearization of (2.4a) and (2.4b) around the steady state, namely

$\lambda U=U^{\prime \prime}+3 c^{2} R_{s}^{-4} U+\left(\mu+\Gamma\left\langle R_{s}^{2}\right\rangle-3 R_{s}^{2}\right) U+2 \Gamma\left\langle R_{s} U\right\rangle R_{s}, \quad U(x+1)=U(x)$,

possesses the zero eigenvalue. The relation between the eigenfunctions of both problems is obtained by linearizing (2.2) and (2.6), which can also be written as $A_{s}+X=\left(R_{s}+U\right) \mathrm{e}^{\mathrm{i}\left(\theta_{s}+\Theta\right)}$ and $\left(R_{s}+U\right)^{2}\left(\theta_{s}^{\prime}+\Theta^{\prime}\right)=c$. It follows that

$$
X=\left(U+\mathrm{i} R_{s} \Theta\right) \mathrm{e}^{\mathrm{i} \theta_{s}} \quad \text { with } 2 U \theta_{s}^{\prime}+R_{s} \Theta^{\prime}=0 .
$$

Using (2.26) to relate the eigenfunctions of (2.14)-(2.15) and (2.25), we shall prove that if (2.25) has a strictly positive eigenvalue, then (2.14)-(2.15) also has a positive eigenvalue. But (2.25) is a scalar equation and its analysis is much simpler.

Lemma 2.12. Let $R_{s}>0$ be a nonconstant solution of (2.4a) and (2.4b). If (2.25) has an eigenvalue such that $\lambda>0$, then (2.14)-(2.15) also exhibits a strictly positive eigenvalue. 
Proof. Let $U$ be an eigenfunction of (2.25), with $\lambda>0$. Defining $X$ as in (2.26) and using (2.3), (2.6), and (2.25), we obtain (after some algebra)

$\lambda U \mathrm{e}^{\mathrm{i} \theta_{s}}=X^{\prime \prime}+\left(\mu+\Gamma\left\langle\left|A_{s}\right|^{2}\right\rangle-2\left|A_{s}\right|^{2}\right) X-A_{s}^{2} \bar{X}+\Gamma\left\langle\bar{A}_{s} X+A_{s} \bar{X}\right\rangle A_{s}$,

$X(x+1)=X(x) \mathrm{e}^{\mathrm{i} v}$

which (multiplying (2.27) by $\bar{X}$ and proceeding as usual) implies that $\mathcal{A}(X)=-\lambda\left\langle U^{2}\right\rangle<0$, and invoking lemma 2.6 we obtain the result.

Lemma 2.13. Let $R_{s}>0$ be a nonconstant solution of $(2.4 a)$ and $(2.4 b)$, with $\Gamma \geqslant 0$. Then (2.25) possesses a strictly positive eigenvalue.

Proof. As in lemma 2.6, the largest eigenvalue of (2.25) satisfies (2.18), with $X$ real and

$\mathcal{A}(X)=\left\langle X^{\prime 2}-3 c^{2} R_{s}^{-4} X^{2}\right\rangle-\left\langle\left(\mu+\Gamma\left\langle R_{s}^{2}\right\rangle-3 R_{s}^{2}\right) X^{2}\right\rangle-2 \Gamma\left\langle R_{s} X\right\rangle^{2}$,

the minimizers being analytic in $\mathbb{R}$. On the other hand, $U=R_{s}^{\prime}$ satisfies $(2.25)$ with $\lambda=0$, as seen by differentiating in (2.3). Thus $\mathcal{A}\left(R_{s}^{\prime}\right)=0$,

$$
\mathcal{A}\left(\left|R_{s}^{\prime}\right|\right)=\mathcal{A}\left(R_{s}^{\prime}\right)+2 \Gamma\left(\left\langle R_{s} R_{s}^{\prime}\right\rangle^{2}-\left\langle R_{s}\left|R_{s}^{\prime}\right|\right\rangle^{2}\right)=-2 \Gamma\left\langle R_{s}\left|R_{s}^{\prime}\right|\right\rangle^{2} \leqslant 0
$$

and since the function $x \rightarrow\left|R_{s}^{\prime}\right|$ is not analytic, it cannot be a minimizer of (2.28). As a consequence, the minimum of (2.28) is strictly negative and using (2.18) it follows that the maximum eigenvalue of (2.25) must be strictly positive. This completes the proof.

Theorem 2.14. Let $A_{s}$ be a steady state of (1.3) with $\Gamma \geqslant 0$ such that $\left|A_{s}\right|$ is nonconstant. Then the steady state is exponentially unstable.

Proof. We must distinguish two cases. If $\left|A_{s}\right|$ vanishes at some $x \in \mathbb{R}$, then according to lemma 2.1(ii) the phase of $A_{S}$ is constant and the result follows from proposition 2.8(i). If instead $R_{s} \equiv\left|A_{s}\right|>0$ in $\mathbb{R}$, then the result is obtained by applying lemmata 2.12 and 2.13 . Thus, the proof is complete.

Remark 2.15. This result in conjunction with our comments at the end of section 1 completely solves the large time dynamics of (1.3) for generic initial conditions (namely, not in the stable manifolds of an unstable steady state) if $\Gamma \geqslant 0$ : each bounded solution converges to an orbitally stable steady state of the form (2.7)-(2.8); these latter steady states are identified in proposition 2.3. If instead $\Gamma<0$ we can only assure that certain steady states (see propositions $2.7,2.8$, and 2.10 ) are exponentially unstable.

\section{The problem (1.7)}

The steady states of (1.7) are given by

$$
A_{s}^{\prime \prime}+\mu A_{s}+\left|A_{s}\right|^{2} A_{s}-\Gamma\left\langle\left|A_{s}\right|^{2}\right\rangle A_{s}=0, \quad A_{s}(x+1)=\mathrm{e}^{\mathrm{i} v} A_{s}(x) .
$$

$A_{s}$ can again be written as in (2.2), with $R_{S}$ and $\theta_{s}$ given by (cf (2.4))

$$
\begin{aligned}
& R_{s}^{\prime \prime}-c^{2} R_{s}^{-3}+\mu R_{s}+R_{s}^{3}-\Gamma\left\langle R_{s}^{2}\right\rangle R_{s}=0, \quad R_{s}(x+1)=R_{s}(x), \\
& \theta_{s}=c \int_{0}^{x} R_{s}^{-2} \mathrm{~d} x+\text { constant }
\end{aligned}
$$


(a)

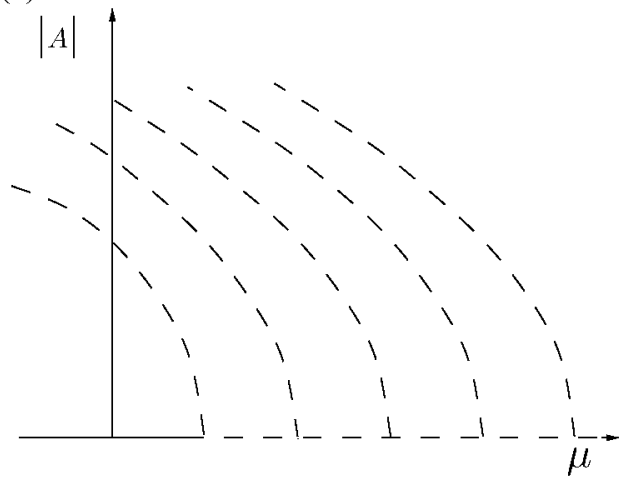

(b)

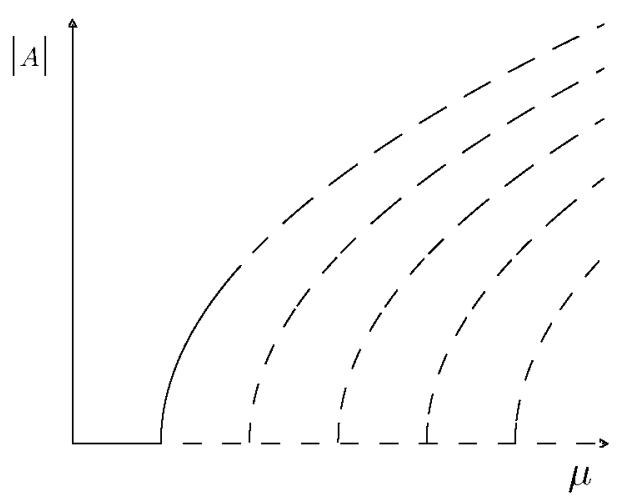

Figure 2. Unstable (- - -) and stable (- spatially uniform SWs of (1.7) for: $(a) \Gamma<1$ and (b) $\Gamma>1$. Plot $(b)$ also applies to the system (1.4)-(1.5) (replacing $A$ by $B$ ) provided that $\gamma_{2}<0$ and $\gamma_{0} \gamma_{1}+\gamma_{2}<0$.

and the constant $c$ given again by (2.5). Using this new equation, the result in lemma 2.1 still holds for this problem. Also, (3.1) exhibits solutions with constant amplitude, of the form (2.7), with $R_{n}$ given by (cf (2.8))

$$
\mu=(v+2 n \pi)^{2}-(1-\Gamma) R_{n}^{2} .
$$

Thus, these families bifurcate from the trivial solution, sub-critically if $\Gamma<1$ and supercritically if $\Gamma>1$. The stability of these steady states is analysed by the dispersion relation (cf (2.12))

$$
\begin{aligned}
& {\left[\lambda-(1-\Gamma) R_{n}^{2}\right]^{2}=(1-\Gamma)^{2} R_{n}^{4} \quad \text { if } m=0, \quad \text { and }} \\
& \left(\lambda+4 m^{2} \pi^{2}-R_{n}^{2}\right)^{2}=16 m^{2} \pi^{2}(\nu+2 n \pi)^{2}+R_{n}^{4} \quad \text { if } m \neq 0 .
\end{aligned}
$$

Using this, we have the following counterpart of proposition 2.3, already obtained by Elmer [7].

Proposition 3.1. If $\Gamma<1$ then all steady states of the form (2.7) are exponentially unstable. If $\Gamma>1$ then these steady states are: (i) orbitally, exponentially stable if $n=0$ and

$$
R_{0}^{2}<2\left(\pi^{2}-v^{2}\right)
$$

and (ii) exponentially unstable if either $n \neq 0$ or $n=0$ and the opposite inequality holds (see figure 2).

Proof. As in proposition 2.3, we only need to use (3.4).

The same argument in the proof of proposition 2.7 gives the following:

Proposition 3.2. Shift $x$ so that $R_{s}$ is an even function of $x$ (which according to lemma 2.1(iii) is always possible). Let the integer $n$, defined in (2.5), be such that $|\nu+2 n \pi|>\pi$. Then, the steady states defined by (2.2), (3.2), and (2.5), with $R_{s} \neq 0$ for all $x$, are exponentially unstable provided that

$$
\left\langle R_{s}^{4}(1-\cos 2 \pi x)\right\rangle+4 \pi\left(\frac{|v+2 n \pi|}{\left\langle R_{s}^{-2}\right\rangle}-\pi\left\langle R_{s}^{2}\right\rangle\right) \geqslant 0 .
$$


Proof. The proof of proposition 2.7 extends verbatim, except for the use of equations (3.1) and (3.2) (instead of equations (2.1) and (2.4)) and the new definition of $\mathcal{A}(\operatorname{cf}(2.17))$ :

$\mathcal{A}(X)=\left\langle\left|X^{\prime}\right|^{2}\right\rangle-\left\langle\left(\mu-\Gamma\left\langle\left|A_{s}\right|^{2}\right\rangle+2\left|A_{s}\right|^{2}\right\rangle|X|^{2}\right\rangle-\frac{\left\langle A_{s}^{2} \bar{X}^{2}+\text { c.c. }\right\rangle-\Gamma\left\langle\bar{A}_{s} X+\text { c.c. }\right\rangle^{2}}{2}$.

Remark 3.3. This result extends the instability result in proposition 3.1 to steady states with nonconstant $\left|A_{s}\right|$. Namely, these steady states are unstable if either (i) $n \neq 0$ or (ii) $n=0$ but $R_{s}$ is sufficiently large.

The result in lemma 2.6 still applies and we have the following counterpart of proposition 2.10:

Proposition 3.4. Any steady state of (1.7) such that $\left|A_{s}\right|$ is not constant and exhibits more than one local maximum in $0<x \leqslant 1$ is exponentially unstable.

Proof. The proof of proposition 2.10 extends verbatim, except for the changes mentioned in the proof of proposition 3.2 .

Finally, the result in theorem 2.14 also applies to (1.7) if $\Gamma \leqslant 0$. More precisely, we have the following theorem.

Theorem 3.5. If $\Gamma \leqslant 0$ then any steady state of (1.7) such that $\left|A_{s}\right|$ is not constant is exponentially unstable.

Proof. We take $X=A_{s}$. Multiplying (3.1a) by $\bar{A}_{s}$, integrating in $0<x<1$, integrating by parts, and substituting $(3.1 \mathrm{~b})$, we obtain

$0=\left\langle\left|X^{\prime}\right|^{2}\right\rangle-\left\langle\left(\mu-\Gamma\left\langle\left|A_{s}\right|^{2}\right\rangle+\left|A_{s}\right|^{2}\right)|X|^{2}\right\rangle=\mathcal{A}(X)+2\left\langle\left(\left|A_{s}\right|^{2}-\Gamma\left\langle\left|A_{s}\right|^{2}\right\rangle\right)|X|^{2}\right\rangle>\mathcal{A}(X)$.

Now, we only need to apply lemma 2.6 (which also holds here) to obtain the stated result.

Remark 3.6. The result above cannot be extended to the case $\Gamma>0$ because in this case Matthews and Cox [18] have found steady states of (1.7) such that $\left|A_{s}\right|$ is not constant and exhibits only one maximum in $0<x \leqslant 1$ (as it must be, see proposition 3.4), in a branch that bifurcates super-critically from the first branch of steady states with constant amplitude. Thus, these steady states with nonconstant amplitude are exponentially stable, at least near threshold.

\section{The problem (1.4)-(1.5)}

If

$$
\gamma_{0} \gamma_{1}+\gamma_{2} \neq 0 \quad \text { and } \quad \gamma_{2}<0
$$

as we assume hereafter, then all results in sections 2 and 3 can be extended to (1.4)-(1.5). For convenience, we use the new variable

$$
\phi=\int_{0}^{x} f(\eta, t) \mathrm{d} \eta
$$


to rewrite (1.4)-(1.5) as

$$
\begin{aligned}
& B_{t}=B_{x x}+\mu B-\gamma_{0}|B|^{2} B-\phi_{x} B, \quad \phi_{t}=\gamma_{1} \phi_{x x}-\gamma_{2}\left(|B|^{2}\right)_{x}, \\
& B(x+1, t)=\mathrm{e}^{\mathrm{i} \nu} B(x, t), \quad \phi(x+1, t)=\phi(x, t), \quad\langle\phi\rangle=0,
\end{aligned}
$$

where the condition $\langle\phi\rangle=0$ is imposed to avoid the spurious symmetry $\phi \rightarrow \phi+$ constant.

The steady states of (4.2)-(4.3) are given by

$$
B_{s}=\left(\frac{\gamma_{1}}{\left|\gamma_{0} \gamma_{1}+\gamma_{2}\right|}\right)^{1 / 2} A_{s}, \quad \phi_{s}^{\prime}=\frac{\gamma_{2}\left(\left|B_{s}\right|^{2}-\left\langle\left|B_{s}\right|^{2}\right\rangle\right)}{\gamma_{1}},
$$

where $A_{s}$ satisfies (2.1) if $\gamma_{0} \gamma_{1}+\gamma_{2}>0$ and (3.1) if $\gamma_{0} \gamma_{1}+\gamma_{2}<0$, with

$$
\Gamma=\frac{\gamma_{2}}{\gamma_{0} \gamma_{1}+\gamma_{2}} \text {. }
$$

Using these, (2.7)-(2.8) and (3.3), the steady states with constant amplitude are

$$
B=R_{n} \mathrm{e}^{\mathrm{i}(v+2 n \pi)}, \quad \phi=0, \quad \text { for } n= \pm 1, \pm 2, \ldots,
$$

where $R_{n}$ is given by

$$
\mu=(v+2 n \pi)^{2}+\gamma_{0} R_{n}^{2}
$$

Note that the bifurcation is super-critical (resp., sub-critical) if $\gamma_{0}>0$ (resp., $\gamma_{0}<0$ ).

The linear stability of a steady state, $\left(B_{s}, \phi_{s}\right)$, is analysed replacing in (4.2)-(4.3) $B-B_{s}$ and $\phi-\phi_{s}$ by $X(x) \mathrm{e}^{\lambda t}+\bar{Y}(x) \mathrm{e}^{\bar{\lambda} t}$ and $\Phi(x) \mathrm{e}^{\lambda t}+$ c.c., respectively, and linearizing. As above, the resulting problem only exhibits real eigenvalues and (setting $Y=\bar{X}$ ) reduces to

$$
\begin{aligned}
& \lambda X=X^{\prime \prime}+\left(\mu-2 \gamma_{0}\left|B_{s}\right|^{2}-\phi_{s}^{\prime}\right) X-\gamma_{0} B_{s}^{2} \bar{X}-\Phi^{\prime} B_{s}, \\
& \lambda \Phi=\gamma_{1} \Phi^{\prime \prime}-\gamma_{2}\left(\bar{B}_{s} X+B_{s} \bar{X}\right)^{\prime} \\
& X(x+1)=X(x) \mathrm{e}^{\mathrm{i} v}, \quad \Phi(x+1)=\Phi(x) .
\end{aligned}
$$

Thus, instability sets in only as $\lambda=0$.

The stability of the steady states (4.6) is considered in the following result (see figures 1 and 2), already obtained by Matthews and Cox [18].

Proposition 4.1. If $\gamma_{0} \gamma_{1}+\gamma_{2}>0$ (resp., $\left.\gamma_{0} \gamma_{1}+\gamma_{2}<0\right)$, then the steady states (4.6) are orbitally exponentially stable if either $n=0$ or $n \neq 0$ and $R_{n}^{2}>2 \gamma_{1}\left[(\nu+2 n \pi)^{2}-\pi^{2}\right] /\left|\gamma_{0} \gamma_{1}+\gamma_{2}\right|$, (resp., $n=0$ and $\left.R_{0}^{2}<2 \gamma_{1}\left(\pi^{2}-v^{2}\right) /\left|\gamma_{0} \gamma_{1}+\gamma_{2}\right|\right)$ and (ii) exponentially unstable if $n \neq 0$ and $R_{n}^{2}<2 \gamma_{1}\left[(v+2 n \pi)^{2}-\pi^{2}\right] /\left|\gamma_{0} \gamma_{1}+\gamma_{2}\right|$ (resp., either $n=0$ and $R_{0}^{2}>2 \gamma_{1}\left(\pi^{2}-v^{2}\right) /\left|\gamma_{0} \gamma_{1}+\gamma_{2}\right|$, or $n \neq 0$ ).

Proof. As in the proofs of propositions 2.3 and 3.1, the dispersion relation is obtained in closed form, and the result follows.

As in section 2, the maximum eigenvalue of (4.8)-(4.10) is given by (cf (2.18))

$$
\lambda_{M}=-\min \left\{\frac{\mathcal{B}(X, \Phi)}{\left\langle|X|^{2}-\Phi^{2} /\left(2 \gamma_{2}\right)\right\rangle}:(0,0) \neq(X, \Phi) \in E\right\},
$$

where $E=\left\{(X, \Phi) \in H^{1} \times H^{1}: X(x+1)=\mathrm{e}^{\mathrm{i} v} X(x), \Phi(x+1)=\Phi(x)\right.$ for all $\left.x,\langle\Phi\rangle=0\right\}$ and

$$
\begin{gathered}
\mathcal{B}(X, \Phi)=\left\langle\left|X^{\prime}\right|^{2}-\frac{\gamma_{1} \Phi^{2}}{2 \gamma_{2}}\right\rangle-\left\langle\left(\mu-2 \gamma_{0}\left|B_{s}\right|^{2}-\phi_{s}^{\prime}\right)|X|^{2}\right\rangle \\
+\frac{\gamma_{0}\left\langle\bar{B}_{s}^{2} X^{2}+\text { c.c. }\right\rangle}{2}+\left\langle\Phi^{\prime}\left(\bar{B}_{s} X+\text { c.c. }\right)\right\rangle
\end{gathered}
$$


We have the following counterpart of lemma 2.6, which is again the key ingredient to extend the instability results in sections 2 and 3 .

Lemma 4.2. Let $\left(B_{s}, \phi_{s}\right)$ be a steady state of $(4.2)-(4.3)$. If $(X, \Phi) \in E$ is such that either (i) $\mathcal{B}(X, \Phi)<0$, or (ii) $(X, \Phi)$ is not an eigenfunction of (4.8)-(4.10) (which happens in particular if either $X$ or $\Phi$ is not analytic) and $\mathcal{B}(X, \Phi)=0$, then (4.8)-(4.10) has a strictly positive eigenvalue.

Proof. The argument in the proof of lemma 2.6 applies again.

Using this we can prove the following result, already obtained by Norbury et al [26, lemma 3] for steady states with constant phase, which allows us to extend to (4.8)-(4.10) all instability results in sections 2 and 3.

Lemma 4.3. Let $\left(B_{s}, \phi_{s}\right)$ be a steady state of (4.2)-(4.3), and let $A_{s}$ defined by (4.4a) be the associated steady state of (1.3) (resp., (1.7)) if $\gamma_{0} \gamma_{1}+\gamma_{2}>0$ (resp., if $\gamma_{0} \gamma_{1}+\gamma_{2}<0$ ), with $\Gamma$ as defined in (4.5). If $A_{s}$ is exponentially unstable, then $\left(B_{s}, \phi_{s}\right)$ is also exponentially unstable.

Proof. Since $A_{s}$ is exponentially unstable, the linearized eigenvalue problem (2.14)-(2.15) possesses a strictly positive eigenvalue, with an eigenfunction $Y$ such that $\mathcal{A}(Y)<0$, where $\mathcal{A}$ is as defined in (2.17) (resp., (3.6)). Substituting (4.4)-(4.5),

$$
X=Y, \quad \text { and } \quad \Phi^{\prime}=\frac{\gamma_{2}\left[\bar{B}_{s} X+\text { c.c. }-\left\langle\bar{B}_{s} X+\text { c.c. }\right\rangle\right]}{\gamma_{1}}
$$

into the right-hand side of (4.11), we obtain $\mathcal{B}(Y, \Phi)=\mathcal{A}(Y)<0$. Now, we only need to apply lemma 4.2 to obtain the stated result.

Using this and the instability results in sections 2 and 3, we have the following theorem.

Theorem 4.4. A steady state of (4.2)-(4.3) such that $\left|B_{s}\right|$ is not constant is exponentially unstable in each of the following cases:

(i) $\left|B_{s}\right|>0$ is not constant and exhibits more than one local maximum in $0 \leqslant x<1$.

(ii) $\gamma_{0} \gamma_{1}+\gamma_{2}>0$ and one of the following conditions holds:

(ii-1) $B_{s}$ exhibits a constant phase (namely, $B_{S}=R_{S}(x) \mathrm{e}^{\mathrm{i} c}$, with $c=$ constant and $R_{s}$ not necessarily of constant sign, which requires that $\nu=0$ or $\pi$ ).

(ii-2) $B_{s}=R_{s} \mathrm{e}^{\mathrm{i} \theta_{s}}$, with $\theta_{s}$ nonconstant and

(ii-3) $B_{s}=R_{s} \mathrm{e}^{\mathrm{i} \theta_{s}}$, with $\theta_{s}$ nonconstant and

$$
\left\langle R_{s}^{2} R_{s}^{2}\right\rangle>\frac{\gamma_{0} \gamma_{1}\left[\theta_{s}(1)-\theta_{s}(0)\right]^{2}}{\left(\gamma_{0} \gamma_{1}+\gamma_{2}\right)\left\langle R_{s}^{-2}\right\rangle^{2}} .
$$

$$
\begin{aligned}
& \qquad \frac{\gamma_{0} \gamma_{1}+\gamma_{2}}{\gamma_{1}}\left\langle R_{s}^{4}(1-\cos 2 \pi x)\right\rangle+4 \pi\left(\frac{|\nu+2 n \pi|}{\left\langle R_{s}^{-2}\right\rangle}-\pi\left\langle R_{s}^{2}\right\rangle\right) \geqslant 0, \\
& \text { provided that }|\nu+2 \pi|>\pi .
\end{aligned}
$$

Proof. The result follows by applying lemma 4.3 and propositions $2.8,2.10,3.2$ and 3.4.

\section{Remarks 4.5.}

(A) Since $\gamma_{2}<0$ (see (4.1)), $\Gamma$ and $\gamma_{0} \gamma_{1}+\gamma_{2}$ exhibit opposite signs (see (4.5)) and the stronger instability results in theorems 2.14 and 3.5 cannot be extended to the system (4.2)-(4.3). 
(B) In the sub-critical case with $\gamma_{0} \gamma_{1}+\gamma_{2}<0$, Matthews and Cox [18] and Norbury et al [26] have found orbitally, exponentially stable steady states of (4.2)-(4.3) such that $\left|B_{s}\right|$ is not constant and exhibits only one maximum in $0<x \leqslant 1$.

(C) The result in part (ii-1) was already obtained in [26] in the limit $|\mu| \gg 1$. The results in parts (i) and (ii-1) show that the 'type II' and 'type III' solutions found in [26], and those in [18, figures 6 and 7] are all unstable. The results (i), (ii-2), and (ii-3) would be useful to ensure the instability of many steady states with varying amplitude and phase, which unfortunately were not considered in [26].

\section{Concluding remarks}

We have analysed the stability of the steady states of some problems involving the GinzburgLandau equation with real coefficients, which are summarized here for convenience.

For the nonlocal equation (1.3), we have shown that all steady states such that $|A|$ is nonconstant are exponentially unstable if $\Gamma \geqslant 0$ (theorem 2.14). This result solves an open problem for the standard, local ( $\Gamma=0)$ Ginzburg-Landau equation with real coefficients. For $\Gamma<0$ we have still obtained instability of the steady states such that either (a) $\left|A_{s}\right|$ exhibits more than one local maximum in $0<x \leqslant 1$ (proposition 2.10), or (b) the phase of $A_{s}$ is constant (proposition 2.8(i)), or (c) $\left|A_{s}\right|>0$ and the phase of $A_{s}$ does not vary much compared with the variation of $\left|A_{s}\right|$ (proposition 2.8(ii)), or (d) $|\nu+2 \pi|>\pi$ and $|A|$ is not too large (proposition 2.7). For the nonlocal equation (1.7), as a main result, we have again shown that all steady states such that $|A|$ is nonconstant are unstable if $\Gamma \leqslant 0$ (theorem 3.5). If $\Gamma>0$ we have still shown that the steady states with nonconstant $|A|$ are still unstable provided that either (a) $|A|$ exhibits at least two maxima in $0 \leqslant x<1$ (proposition 3.4), or (b) $\mid$ phase of $A(1)$ - phase of $A(0) \mid>\pi$, provided that $|A|$ is not too small (proposition 3.2). With these, we have completed previous instability results on (a) constant-amplitude steady states [7] and (b) amplitude-modulated steady states in the particular cases in which (b-1) the nonlocal term is absent $(\Gamma=0)$ and the domain is laterally unbounded [25] and (b-2) $v=0$ or $\pi$, the steady state exhibits constant phase and $|\mu| \gg 1$ [26].

For the problem (1.4)-(1.5), with $\gamma_{2}<0$, we have shown (theorem 4.4) that the steady states with nonconstant $|B|$ are unstable if $|B|$ exhibits more than one maximum in $0<x \leqslant 1$. If, in addition, $\gamma_{0} \gamma_{1}+\gamma_{2}>0$, then the steady states with nonconstant $|B|$ are still unstable provided that either they exhibit a constant or almost constant phase, or $\mid$ phase of $B(1)$-phase of $B(0) \mid>\pi$ and $|B|$ is not too small. These results complete previous instability results on the constant amplitude steady states [18] and the amplitude-modulated steady states with constant phase for $|\mu| \gg 1$ [26].

\section{Acknowledgments}

I am indebted to Dr Francisco Mancebo for a critical revision of the manuscript and for some useful comments. This research was partially supported by the Spanish Ministry of Science and Technology (Grant MTM2004-03808) and NASA (Grant NNC04GA47G).

\section{References}

[1] Newell A and Whitehead J 1969 Finite bandwith, finite amplitude convection J. Fluid Mech. 38 279-304

[2] Cross M C and Hohenberg P C 1993 Pattern formation outside of equilibrium Rev. Mod. Phys. 65 851-1112

[3] Knobloch E and De Luca J 1990 Amplitude equations for travelling wave convection Nonlinearity 3 55-73 
[4] Alvarez-Pereira C and Vega J M 1992 On the pulsating instability of two-dimensional flames Eur. J. Appl. Math. $355-73$

[5] Vega J M 1993 On the amplitude equations arising at the onset of the oscillatory instability in pattern formation SIAM J. Math. Anal. 24 603-17

[6] Martel C and Vega J M 1996 Finite size effects near the onset of the oscillatory instability Nonlinearity 9 1129-71

[7] Elmer F J 1988 Nonlinear and nonlocal dynamics of spatially extended systems: stationary states, bifurcations and stability Physica D $30321-42$

[8] Faraday M 1831 On the forms and states assumed by fluids in contact with vibrating elastic surfaces Phil. Trans. R. Soc. Lond. $121319-40$

[9] Lord Rayleigh J W S 1883 On the crispations of fluid resting upon a vibrating support Phil. Mag. 16 50-8

[10] Miles J and Henderson D 1990 Parametrically forced surface waves Ann. Rev. Fluid Mech. 22 143-65

[11] Fauve S 1995 Parametric instabilities Dynamics of Nonlinear and Disordered Systems ed G Martínez Mekler and T H Seligman (Singapore: World Scientific) pp 67-115

[12] Mancebo F J and Vega J M 2004 Viscous Faraday waves in 2D large aspect ratio containers Preprint

[13] Martel C, Knobloch E and Vega J M 2000 Dynamics of counterpropagating waves in parametrically forced systems Physica D 137 94-123

[14] Mancebo F J and Vega J M 2004 Standing wave description of nearly conservative, parametrically driven waves in extended systems Physica D $197346-63$

[15] Vega J M, Knobloch E and Martel C 2001 Nearly inviscid Faraday waves in annular containers of moderately large aspect ratio Physica D $154313-36$

[16] Lapuerta V, Martel C and Vega J M 2002 Weakly-dissipative Faraday waves in 2D large aspect ratio annuli Physica D 173 178-203

[17] Coullet P and Iooss G 1990 Instabilities of one-dimensional cellular patterns Phys. Rev. Lett. 64 866-9

[18] Matthews P C and Cox S M 2000 Pattern formation with a conservation law Nonlinearity 13 1293-320

[19] Matthews P C and Cox S M 2001 New instabilities in two-dimensional rotating convection and magnetoconvection Physica D 149 210-29

[20] Weidman P D, Linde H and Velarde M G 1992 Evidence of solitary wave behavior in Marangoni-Bénard convection Phys. Fluids A 4 921-6

[21] Golovin A A, Nepomnyashkchy A A and Pismen L M 1993 Interaction between short-scale Marangoni convection and long-scale deformational instability Phys. Fluids $634-48$

[22] Plaut E and Busse F H 2002 Low Prandtl number convection in a rotating cylindrical annulus J. Fluid Mech. $464345-63$

[23] Tanaka D and Kuramoto Y 2003 Complex Ginzburg-Landau equation with nonlocal coupling Phys. Rev. E 68 026219

[24] Kramer L and Zimmermann W 1985 On the Eckhaus instability for spatially periodic patterns Physica D 16 221-32

[25] Doelman A, Gardner R A and Jones C K R T 1995 Instability of quasiperiodic solutions of the Ginzburg-Landau equation Proc. R. Soc. Edinb. A 125 501-17

[26] Norbury J, Wei J and Winter M 2002 Existence and stability of singular patterns in a Ginzburg-Landau equation coupled with a mean field Nonlinearity 15 2077-96

[27] Henry D 1989 Geometric Theory of Semilinear Parabolic Equations (Berlin: Springer)

[28] Ladyženskaja O A, Solonnikov V A and Ural'ceva N N 1968 Linear and Quasilinear Equations of Parabolic Type (Providence, RI: American Mathematical Society)

[29] Adams R A 1975 Sobolev Spaces (New York: Academic)

[30] Hale J K 1989 Asymptotic Behavior of Dissipative Systems (Providence, RI: American Mathematical Society)

[31] Coddington E A and Levinson N 1955 Theory of Ordinary Differential Equations (New York: McGraw-Hill)

[32] Dieudonné J 1960 Foundations of Modern Analysis (New York: Academic)

[33] Courant R and Hilbert D 1962 Methods of Mathematical Physics vol I and II (New York: Wiley, Interscience)

[34] Kreyszig E 1978 Introductory Functional Analysis with Applications (New York: Wiley) 